\title{
PID Parameters Tuning Based on a Self-Adaptive Immunity Ant Colony Algorithm
}

\author{
Yuying Shao, Zhengquan Lv \\ Shanghai municipal electric power company \\ Shanghai, China
}

\author{
Li Deng, Aiping Wang \\ School of Mechatronical Engineering and Automation \\ Shanghai University \\ Shanghai, China
}

\begin{abstract}
For the disadvantages of being easy to fall into premature of particle swarm optimization and the lengthy process of immune algorithm, putting the immunization information processing mechanisms of the immune system into PSO algorithm. A particle swarm algorithm based on immune selection (IPSO) was proposed, and its application to the parameter tuning and self-adaption in PID controllers, and designing the PID controller parameters. Simulating by MATLAB, experimental results showed that the algorithm can solve the problem of PSO algorithm premature convergence, and applying to self-tuning PID controller.
\end{abstract}

Keywords- Particle swarm optimization; Immune algorithm; IPSO; PID Parameters Tuning

\section{INTRODUCTION}

In industrial process control, PID control with the advantages of simple, robustness strength is widely used in various areas. According to statistics, the proportion of the PID control strategies in actual control system is more than $90 \%$, and the tuning and adaption of the PID controller parameters have always been the most important problem. Ziegler and Nichols designed out of the formula of Z-N in 1942, this is the first method of PID parameter tuning, and so far it has found wide application in various industrial control applications. Then, in 1953, Cohen and Coon inherited and developed the Z-N formula and designed the Cohen-Coon tuning formula of the size of the time delay of considering a controlled process [1].

Conventional PID controller structures is not yet perfect, the widespread presences of integral saturated, overshoot, and the disadvantage of the instability of the system caused by the sensitivity of differential effects to high-frequency interference. Another problem with traditional PID control is not unable to make target tracking performances and interference suppression characteristics be optimal at the same time but the new developments are introducing the adaptive control, optimal control, predictive control, robust control and intelligent control strategies into a new kind of PID control in the traditional PID control [2].

Reference [3] attempted to introduce the PSO algorithm to PID control, the PSO algorithms is evolved from the Bionic optimization algorithms, and the computation is simple and good optimization effect. But the PSO algorithm is easy to fall into premature etc. weakness. In order to solve this problem, an immune algorithm is introduced. The immune algorithm with characteristics of immune and genetic characteristics can help prevent particle swarm into premature, while a combination of both avoids the disadvantages of the lengthy process of the immune algorithm.

\section{PROBLEM DESCRIPTION}

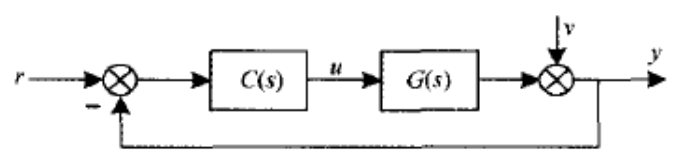

Figure 1. A closed-loop feedback control system of PID controller

The PID controller is a cross control algorithm based on the information estimations of the variable of " past," "now" and "future" .The system is mainly composed of the controlled object and the PID controller two parts, as shown in figure 1. $C(\mathrm{~s})$ is a standard PID controller, and $G(\mathrm{~s})$ is controlled object.

The Parameters tuning of PID controller and selfadaption refer to after according to the system model and the all kinds of performance indexes and on the condition of identifying the controller as the formation of PID, using various methods to tune the parameters of PID controller to meet the desired requirements and achieve the desired control goals of the dynamic characteristics and static characteristics of controlled loop composed of control objects, controller, actuator and the feedback elements.

\section{PARTIClE SWARM OPTIMIZATION}

In recent years, Particle Swarm Optimization (particle swarm optimization, PSO), as a new random evolutionary computation methods, is widely used because of the characters of being simple common, suitable for parallel processing and the low requirements of the objective function and constraints. But because the PSO algorithm is based on the all particles and the search of their own experience towards the direction of the optimal solution to "fly", which will result in particles swarm diversity decline in the evolutionary process, so when the algorithm converges is up to a certain precision, which cannot continue to optimize and easily get into local optimization, resulting in poor accuracy which can be achieved [4].

The iteration update formula of its particles is shown as equation (1) . 


$$
\left\{\begin{array}{l}
V_{i}^{n+1}=\omega V_{i}^{n}+a_{1} \text { rand }()\left(P_{b e s t}-X_{i}^{n}\right)+a_{2} \text { rand }\left(G_{b e s t}-X_{i}^{n}\right) \\
X_{i}^{n+1}=X_{i}^{n}+V_{i}^{n+1}
\end{array}\right.
$$

Where: $\mathrm{n}$ is the number of iterations, $\omega$ is inertia weight, $\mathrm{a}_{1}$ and $\mathrm{a}_{2}$ are reading factors, rand () is random numbers evenly distributed over $(0,1)$.

\section{IMMUNE ALGORITHM}

Immune algorithm is algorithm that is pioneered on getting inspired from Human Bionics by scientists [5], in the field of life sciences; people have widely and depth of studies on the natural phenomena of heredity and immunity and other. The immune algorithm from the evolution of the genetic algorithm is almost the same with the genetic algorithm, and only a more used immune function is used, and it is a variant of the genetic algorithm, which is not using hybrids but using the method of an injected vaccine. Vaccines are good genes within a chromosome, and inoculating to other chromosomes.

\section{IPSO ALGORITHM}

\section{A. The basic principle of the IPSO algorithm}

The Particle swarm optimization (IPSO) based on immune selection introduces the immunization information processing mechanisms into the immune system in PSO algorithm, the problems waiting for solving are seen as antigen, and each antibody can represent a solution to the problem, and each antibody is simultaneously a particle in a particle swarm. The Antigen and antibody affinities are measured by the adaption of the PSO algorithm, reflecting on the satisfaction degree of the objective functions and their constraint conditions; the antibody affinity reflects the difference between the particles, that is, the species diversity.

Compared with the standard PSO algorithm, the immune Particle swarm optimization is composed of 3 key components, the basic PSO algorithm, can be run independently, used to control the iterative processes of the whole algorithm; the immune memory and function of selfregulation, to ensure the diversity of populations; implementation of vaccination, for improving the convergence performance [6].

\section{B. The process of the IPSO algorithm}

The Particle Swarm Optimization based on immune selection (IAPSO) algorithm procedures are as follows:

Step 1 initializing the PSO: randomly generating the positions and velocities of $N$ particles in the permitted scale, calculating adaptive values to get the of individual extreme values and global extremes of the initialize group;

Steps 2 producing the vaccines: acting the global optimization particles as an immune "vaccine";

Step 3 updating the positions and velocities of the particles: updating the velocity and position of each particle according to type (1);
Step 4 groups updating: Comparing with the adaptive values of the updating particles and the former. If the adaptive value increases, and the position of the particle can only be updated, or retaining the original position of the particle;

Step 5 the new particles of random production: in order to increase the diversity of groups, there are $M$ particles randomly producing during each iteration process and calculating the adaptive values;

Step 6 probability choices of $\mathrm{N}$ particles to form a new group: the new group formatting from the above $N+M$ particles, randomly selecting $N$ particles according to (2) forms a new group [7];

$$
P_{i}=\frac{\sum_{j=0}^{r+t}\left|f\left(x_{i}\right)-f\left(x_{j}\right)\right|}{\sum_{i=0}^{r+t} \sum_{j=0}^{r+t}\left|f\left(x_{i}\right)-f\left(x_{j}\right)\right|}
$$

Step 7 the immunization and immune selections: using steps 2 produces immune "vaccine" to vaccinate for $R$ particle in Group, calculating the adaptive value for particles of vaccination and the immune selection according to the adaptive value. If the adaptive value reduces, the particle is retained, or cancels the vaccine. This contributes to a new generation of groups;

Step 8 Updating the individuals and extreme values of global groups: updating the individual and extreme values according to the adaptive values of the new generation of group;

Step 9 determining whether satisfying the constraints: If the iteration count is greater than the maximum number of iterations, the ending loop output, otherwise going to step 2.

\section{The Parameters Of The PID Controller Design}

The PID controller tuning parameters using the Particle swarm optimization based on immune selection must establish comprehensive fitness functions, evaluating the advantage and disadvantage of the performances of particles and acting as the updating evidences of the individual best and the global optimization particles, making initial solution gradually to the evolution of optimal solution. Usually, a set of good PID control parameters of $K_{p}, K_{i}$ and $K_{d}$ will produce a good step response curve and the smallest timedomain performance indicators. However, the time-domain performance indicators include: adjusting time $t_{s}$, rising time $t_{r}$, maximum overshoot Mp and steady state errors $e_{s s}$. Fitness function defined in this article is ITEA [8], shown as equation (3).

$$
J=I T A E=\int_{0}^{\infty} t e(t) d t
$$




\section{THE SIMULATION EXPERIMENT}

Choosing 2 systems of 1 and 2, its passing functions as (4) and (5) shown, using Particle swarm optimization based on immune selection for iterative calculations, and comparing to the simple PSO optimize algorithm and simulation using MATLAB software, the program selection algorithm parameters are as follows: Numbers of particles $N=50$; Learning factors $\mathrm{a}_{1}=\mathrm{a}_{2}=2.0$; Values of $\omega$ between 0.4 to 0.9 , and as the number of iterations increases linearly with decreases; the number of iterations MAX $=100$.

$$
\begin{gathered}
G_{1}(s)=\frac{e^{-0.5 s}}{s+1} \\
G_{2}(s)=\frac{5(s+2)(6.3 s+1.8)}{(s+5)(s+4)\left(s^{3}+3 s^{2}+5\right)}
\end{gathered}
$$

The dynamic response curve of $G_{1}(\mathrm{~s})$ is shown in Figure 2. The dotted lines are the response curves for PSO algorithm; the solid lines are the response curves for a given choice of immune Particle swarm optimization (IPSO).

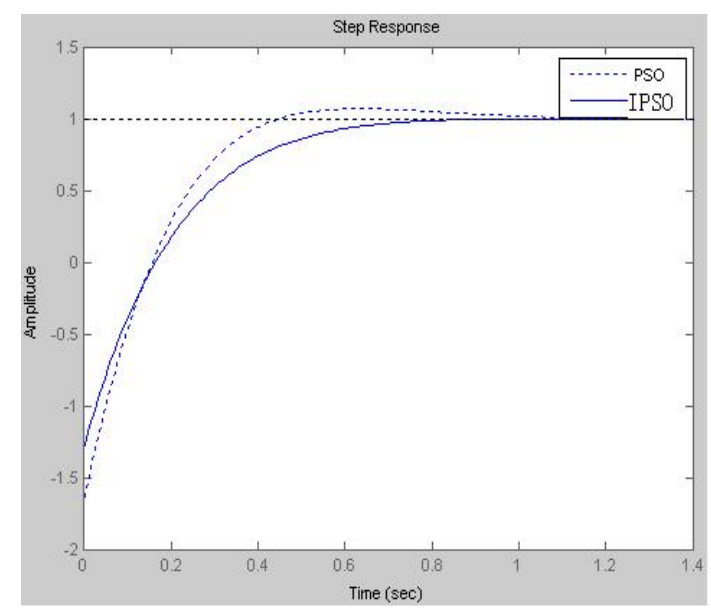

Figure 2. The dynamic response curve of $G_{1}(\mathrm{~s})$

The dynamic response curve of $G_{1}(\mathrm{~s})$ is shown in Figure 2. The dotted lines are the response curves for PSO algorithm; the solid lines are the response curves for a given choice of immune Particle swarm optimization (IPSO).

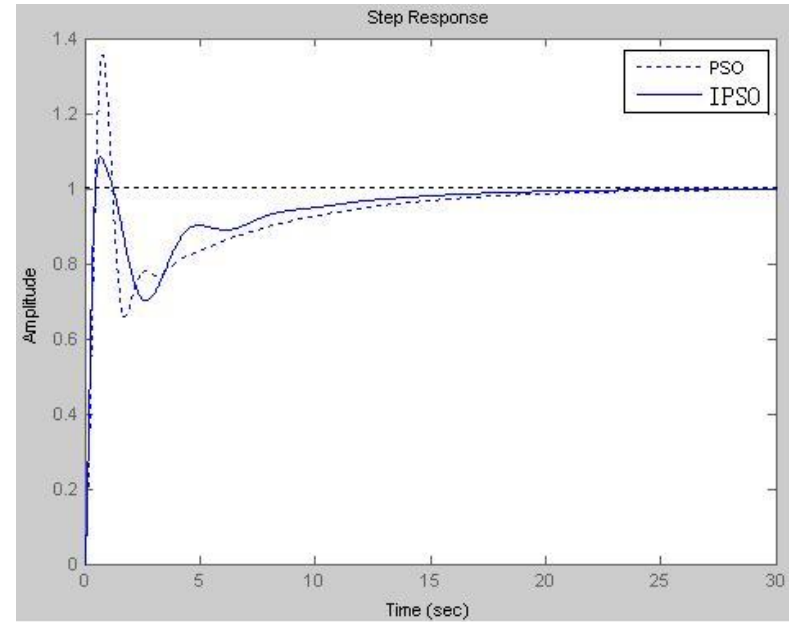

Figure 3. The dynamic response curve of $G_{2}(\mathrm{~s})$

As can be seen from the Fig. 2 and Fig. 3 the lagging system PID controllers is easier to stabilize, followed by Fig. 2 shown , the higher order, the more difficult to calculate a better controller parameters, as shown in Fig.3. The 2 systems eventually reach a steady state after the PID controller adjusting and then getting feedback again. Fig. 2 and Fig.3 show that simply using PSO algorithm isn't as good as the algorithm presented in this article.

The calculation results and the dynamic behaviors of the 2 systems are shown in Table 1, the parameters of the PID controller are calculated from the PSO algorithm and the algorithm proposed in this paper, respectively.

TABLE I. CALCULATION RESULTS AND THE DYNAMIC BEHAVIORS OF THE 2 SYSTEMS

\begin{tabular}{|c|c|c|c|c|c|c|c|c|}
\hline $\begin{array}{c}\text { syst } \\
\mathbf{e m}\end{array}$ & $\begin{array}{c}\text { meas } \\
\mathbf{u r e}\end{array}$ & $\mathbf{K}_{\mathbf{d}}$ & $\mathbf{K}_{\mathbf{p}}$ & $\mathbf{K}_{\mathbf{i}}$ & $\mathbf{t}_{\mathbf{r}}(\mathbf{s})$ & $\mathbf{t}_{\mathbf{p}}(\mathbf{s})$ & $\mathbf{t}_{\mathbf{s}}(\mathbf{s})$ & $\boldsymbol{\sigma}(\mathbf{\%})$ \\
\hline \multirow{2}{*}{1} & PSO & 0.62 & 3.56 & 2.85 & 0.45 & 0.67 & 0.78 & $6.86 \%$ \\
\cline { 2 - 9 } & IPSO & 0.56 & 2.94 & 2.30 & 1.01 & 1.12 & 0.56 & $0.15 \%$ \\
\hline \multirow{2}{*}{2} & PSO & 0.59 & 4.00 & 2.81 & 0.47 & 0.79 & 12.1 & $35.64 \%$ \\
\cline { 2 - 9 } & IPSO & 1.22 & 2.78 & 3.56 & 0.54 & 0.72 & 9.76 & $8.73 \%$ \\
\hline
\end{tabular}

From the table 1 , the adjustable volume and regulation time calculated from the system under the controlling of the controller using the algorithm in this paper are remarkable decreasing compared to the results calculated from the traditional of PSO algorithm. What's more, the $\mathrm{K}_{\mathrm{p}}$ data of the PSO from the system 2, we can see that the particle groups after calculation have flied to the border and into premature, so the algorithm effect used in this work is more better.

\section{CONCLUSION}

For the shortcomings of the conventional PID control methods, this article provides with Particle swarm optimization based on immune selection to tune the PID controller parameters. The Particle swarm optimization based on the immune selection is not only to maintain the fast-track effectiveness of the PSO algorithm and the 
stability of the immune algorithm, and which also can effectively avoid the PSO and immune algorithm dropping into the local solutions. The robustness of immune Particle swarm optimization after the disturbance factors is clearly superior to the PSO and immune algorithm. Simulating by MATLAB, the experimental results showed that this method can reduce the system adjusting time and overshoot, and the system's dynamic performances are significantly improved, and the large inertia, nonlinear and time-varying control system can be good controlled.

\section{REFERENCES}

[1] Lei Wang, Wenzhong Song. PID control [J]. Process Automation Instrumentation, 2004, 254:1-5

[2] ZhiYang, Zhitang Chen, Zhengping Fan, Xiaodong Li. Tuning of PID controller based on improved Particle-Swarm-Optimization [J]. Control Theory \& Applications, 2010,27 (10): 1345-1352.

[3] Jiajun Zhang, Optimization Parameters of PID Controller Parameters Based on Particle Swarm Optimization [J]. Computer Simulation, 2010, 10:191-193.

[4] Jun Feng, yuncan Xue, Jinlong Jiang. Study on A New Modefied Particle swarm optimization [J]. Journal of Hohai University Changzhou, 2006,20 (1): 10-13.
[5] Lei Wang, Jin Pan, Licheng Jiao. The Immune Algorithm [J]. ACTA ELECTRONICA SINICA, 2000,7:74-78.

[6] Jingmin Wu, hongfu Zuo, Yong Chen. A Combined Forecasting Method Based On Particle Swarm Optimization with Immunity Algorithms [J]. Journal of Systems \& Management, 2006,15 (3): 229233.

[7] Xi Chen, Jiafu Jiang . Particle swarm optimizations with Immunity for Traveling Salesman Problems [J]. Computer \& Digital Engineering, 34 (6): 10-12.

[8] Ziqiang Li, Meicheng Xue. Simulating Study of Performance Objectives for PID Parameters Self-tuning [j]. Automation \& Instrumentation, 2009,2:30-33.

[9] Jiao Zhang, Yinya Li, Andong Sheng. Design of a Robust PID Controller for First-order Plus Dead Tim e Processes with Parametric uncertainties [J]. Computer Simulation, the lagging process 2006, 12:174-178.

[10] Kennedy J,Eberhart R. Particle swarm optimization[C]//Proceedings of International Conference on Neural Net works. Perth, Australia: IEEE, 1995:1942-1948.

[11] Gaing Z L. A particle swarm optimization approach for optimum design of PID controller in AVR system[J].IEEE Transactions on Energy Conversion, 2004, 9 (2):384-391.

[12] Shi Y, Eberhart R. A modified particle swarm optimizer[C]/Proceedings of International Conference on Evolutionary Computation. Anchorage, Alaska: IEEE, 1998:69-73. 